\title{
Very, very late stent thrombosis triggered by in-stent neoatherosclerosis: optical coherence tomography findings
}

\author{
Geovana A. João ${ }^{1}$, Carlos Eduardo S. Portela ${ }^{1}$, Luiz F. Ybarra ${ }^{1,2}$, Philippe Généreux ${ }^{3,4}$, Adriano Caixeta ${ }^{1,2}$ \\ ${ }^{1}$ Escola Paulista de Medicina, Universidade Federal de São Paulo, Brazil \\ ${ }^{2}$ Hospital Israelita Albert Einstein, São Paulo, Brazil \\ ${ }^{3}$ Columbia University Medical Center, New York, NY; Cardiovascular Research Foundation, New York, NY, USA \\ ${ }^{4}$ Hôpital du Sacré-Coeur de Montréal, Université de Montréal, Montréal, Quebec, Canada
}

Adv Interv Cardiol 2016; 12, 2 (44): 181-182

DOI: $10.5114 /$ aic. 2016.59373

A 64-year-old woman with dyslipidemia, hypertension and type 2 diabetes plus an existing $4.0-\mathrm{mm} \times$ 28-mm bare-metal stent (BMS; Multilink Penta, Abbott Vascular, US) deployed over 10 years ago in the right coronary artery (RCA) was admitted to our emergency department with inferolateral and posterior ST-segment elevation acute myocardial infarction. Coronary angiography showed occlusion and thrombotic plaque in the mid RCA at the site of the prior stent implantation. After thrombectomy, optical coherence tomography (OCT) documented the presence of in-stent neoatherosclerosis with lipid-laden plaque, mild calcification, clustered macrophages and a disrupted thin-cap fibroatheroma (TCFA), a pattern similar to the morphology seen with typical native atherosclerosis (Figure 1).

In-stent restenosis has widely been considered a stable process, showing an early peak of intimal hyperplasia followed by a stationary period generally after 1 year following stent implantation [1]. More recently, pathologic and in vivo intravascular imaging studies have challenged this finding by showing that lipid-rich plaque with macrophage infiltration and a necrotic core may occur inside the stent. This process of in-stent neoatherosclerosis occurs within months to a few years, compared with the decades that it generally takes for native disease to de- velop [2, 3]. Previous autopsy studies have shown a higher incidence of neoatherosclerosis in lesions treated with first- and second- generation drug-eluting stents (DES) compared with BMS ( $31 \%$ vs. $30 \%$ vs. $16 \%$, respectively) $[2,3]$. Of note, neoatherosclerosis presentation occurred on average at $\sim 400$ days after first-generation DES, $\sim 200$ days after second-generation DES, and $\sim 2,000$ days after BMS [2, 3]. Much like native coronary disease, the infiltration of foamy macrophages within the neointima could possibly cause thinning of the fibrous cap, leading to the development of a TCFA and complications of rupture with thrombosis, as seen in the present case.

The present report describes an unusual case of very, very late stent thrombosis - occurring more than a decade after BMS at 3,710 days - with evidence of neoatherosclerosis. This case highlights the need for continuous and perpetual secondary prevention of coronary artery disease in patients receiving percutaneous coronary intervention. The permanent presence of metallic foreign material in the vessel wall might indefinitely remain a potential trigger for stent failure at extended follow-up.

\section{Conflict of interest}

The authors declare no conflict of interest. 

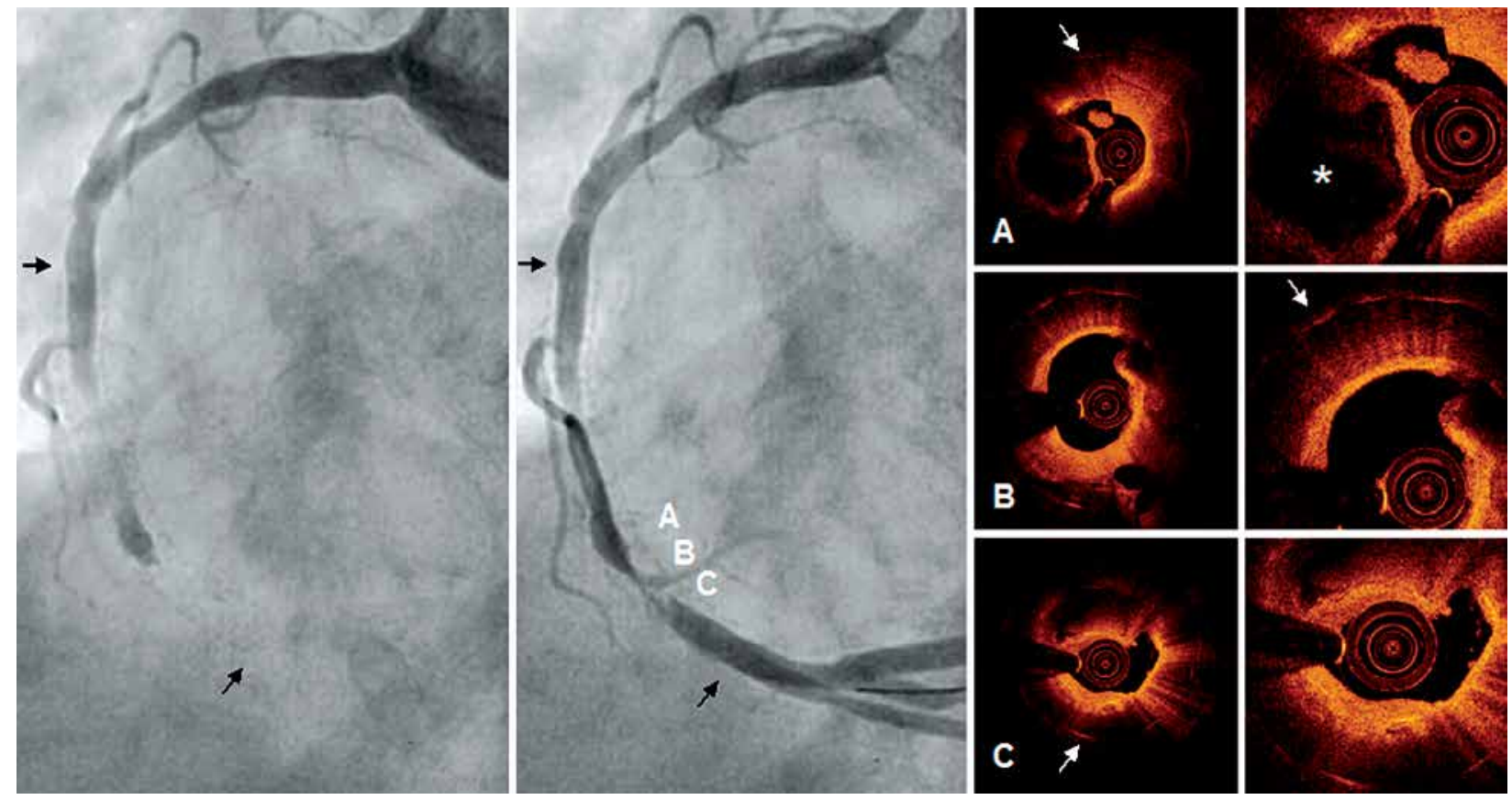

Figure 1. RCA angiography in right anterior oblique view before (left) and after (middle) thrombus aspiration. The region between the arrows represents the region covered by the BMS implanted over a decade earlier. OCT (right) demonstrates that TCFA has developed inside the stent and caused plaque rupture and stent thrombosis. Arrows indicate examples of stent struts. Asterisks indicate the presence of a cavity behind the residual fibrous plaque. The necrotic core is seen as a signal-poor region within the plaque, with poorly delineated borders, a fast signal drop-off, and low OCT backscattering covered by a high intensity signal by the fibrous cap (A-C). Note the presence of cholesterol crystal at 6 o'clock in panel $C$ and white thrombus at 12 o'clock in panel A. A cluster of macrophages with a high signal with dorsal shadowing is seen from 9 to 12 o'clock in panel B. Figures on the right are replicated and zoomed

\section{References}

1. Chen MS, John JM, Chew DP, et al. Bare metal stent restenosis is not a benign clinical entity. Am Heart J 2006; 151: 1260-4.

2. Nakazawa G, Otsuka F, Nakano M, et al. The pathology of neoatherosclerosis in human coronary implants bare-metal and drug-eluting stents. J Am Coll Cardiol 2011; 57: 1314-22.

3. Otsuka F, Vorpahl M, Nakano M, et al. Pathology of second-generation everolimus-eluting stents versus first-generation sirolimus- and paclitaxel-eluting stents in humans. Circulation 2014; 129: 211-23. 